\title{
Evaluation of the application of Clinical Pathways in therapy of Cerebral infarction Patients in China Using the Hospital Database: a Real-world Study
}

Bin Wang ( $\square$ gam_wb@hotmail.com )

China Academy of Chinese Medical Sciences

Ling Xin

Anhui University of Traditional Chinese Medicine

Chen Di

China Academy of Chinese Medical Sciences Guanganmen Hospital

Tao Kong

Dongfang Hospital

Hongwi Zhou

China Academy of Chinese Medical Sciences

Xie Qi

China Academy of Chinese Medical Sciences

Research article

Keywords: Cerebral infarction, Clinical pathways, Real-world study

Posted Date: October 14th, 2019

DOI: https://doi.org/10.21203/rs.2.15993/v1

License: (1) This work is licensed under a Creative Commons Attribution 4.0 International License.

Read Full License 


\section{Abstract}

BACKGROUND A real-world study was performed to evaluate the application of clinical pathways (CP) in treatment of cerebral infarction patients compared with normal medical care. The benefit of clinical pathways application was evaluated by assessing the length of in-hospital stay (LOS) and hospitalization costs in treatment of cerebral infarction patients.

METHODS Data were derived from our hospital database. We enrolled all patients who underwent stroke treatment (International Classification of Diseases, 10th rev. ed., Clinical Modification [ICD- 10-CM] procedure 163.90) between June 1, 2015 and May 31, 2016. The criteria within this evaluation included the average LOS and costs (hospitalization, drugs, laboratory and radiology). Student's t-test and nonparametric Wilcoxon's rank-sum test were performed and statistical significance was set as 0.05 . Results were presented as mean \pm SE.

\section{Background}

Clinical pathway, also known as critical pathway or integrated care pathway, is a clinical approach to link evidence to practice, and thereby optimizes clinical outcomes and maximizes clinical efficiency[1,2]. Clinical pathway has been implemented in many countries, including China[3]. Moreover, clinical pathway is widely used in surgical treatments and has achieved impressive outcome. However, there is no clarified report on the management of internal medicine diseases, such as cerebral infarction [3].

World Health Organization's recent report indicated cerebral infarction is a leading cause of disability and the second common cause of death in adults around the world[4, 5]. It is estimated that about 1.5-2 million of cerebral infarctions occur in China every year [6]. A recent study showed cerebral infarction is the second most common cause of death among both the urban and rural residents in China, which is a unbearable financial burden of patients and the government [6]. Standardized treatment of cerebral infarction can not only reduce infection and complications but also reduce hospital costs among cerebral infarction patients [7]. Therefore, the National Health and Family Planning Commission of China (NHFPC, formerly the Ministry of Health) commissioned a panel of physician experts to come up with more than 1000 drafts of clinical pathways for treatment of kinds of disorders including cerebral infarction.

In order to integrate the characteristics of the diagnosis and treatments of cerebral infarction used in traditional Chinese medicine and in Western medicine, we revised the clinical pathway templates, compiled the data from HIS, LIS, PACS and EMR in our hospital[8], and evaluated its application by assessing LOS and hospital costs in patients with cerebral infarction in our hospital.

\section{Methods}

- Data source 
Data were derived from the Database of the First Affiliated Hospital of Anhui University of Chinese Medicine (Anhui, China). This study was done using a hospital-based composite database stored in the university's hospital information systems (HIS). This database contains all information of cerebral infarction patients, such as age, sex, diagnosis, International Classification of Diseases (ICD) -10 code, surgical history, outpatient/inpatient status, prescriptions, and laboratory data.

- Patient selection and Data retrieval

We enrolled all patients who underwent cerebral infarction treatment (International Classification of Diseases, 10th rev. ed., Clinical Modification [ICD-10-CM] procedure 163.90) between June 1, 2015 and May 31, 2016. These patients were cared for under a clinical pathway as described below. Total hospital costs in this prospective cohort was compared with a historical control group of patients undergoing the same procedure (but without the clinical pathway) in the same department between June 1, 2014 and May 31, 2015. All patients undergoing cerebral infarction treatment were included in each of the two (before and after) groups. Patients with complications were excluded from the study.

Furthermore, we also evaluated changes before and after implementation of the clinical pathway in cost centers directly affected by the pathway, such as the length of hospital stay. Total hospital costs were the sum of fixed and variable costs attributed to hospital departments including patient ward, radiology, pharmacy, laboratory, therapeutic department, and diet- preparation department.

Inclusion, exclusion and withdrawal criteria.

Inclusion criteria

- Adult patients of both genders

- Meeting the criteria for ICD-10, cerebral infarction (I63.9)

Exclusion criteria

- Aged more than 80 years

- Suffering from other serious diseases, such as malignant tumor, diabetes mellitus, digestive tract hemorrhage, liver injury, lung injury, fracture, etc.

- Discharge without doctor's advice or incomplete treatment.

\section{Development of a New Clinical Pathway (Description of Solutions)}

Our hospital implemented the clinical pathway management system in all departments in June 1,2015. A detailed description of the pathway development has been previously reported[9]. A multidisciplinary team consisting physicians, nurses, pharmacists, statisticians and data analysts developed detailed flow charts of treatment processes for patients undergoing cerebral infarction treatment. This team collects all data from patients during hospitalization in order to provide decision support for the construction and optimization of clinical pathways[8]. The final path requires consistent approval from team members. A 
variety of interventions were defined at different stages of treatment (Table 1). After the pathway was initiated, regular meetings were held to assess performance and adjustments were made accordingly.

- Statistical Analysis

Factors in the criteria included the average LOS and costs (hospitalization, drugs, laboratory and radiology). Student's t-test and nonparametric Wilcoxon's rank-sum test were run using SPSS (IBM, Armonk, NY) and statistical significance was set as 0.05 . Results were presented as means \pm SE.

- Ethics

Ethics committee approval was not required for this study. Anonymized data were provided by the university hospital for collecting information on hospitalizations.

\section{Results}

\section{Baseline Characteristics}

Between June 1, 2014 and May 31, 2016, 1533 patients with cerebral infarction were diagnosed and treated in the hospital (Fig 1). The number of hospitalizations was $7.66 \%$ more than the previous year, increased from 444 patients to 478 . Table 1 presents the baseline characteristics of patients. The sex ratio (male/female) for control group and clinical pathway group were 1.59 and 1.70 respectively. The control group reported an average age of $58.79 \pm 13.28$, whereas the clinical pathway group was $60.24 \pm$ 10.77. Neither the $\chi 2$ analysis of the patients' sex nor the Student $t$ test of the patients' age showed a marked difference between the control and clinical pathway group in the cerebral infarction patient population (Table 1).

Table 1. Characteristics of the patients with cerebral infarction, 2014 - 2016

\begin{tabular}{lllll}
\hline & Control Group $(\mathrm{n}=444)$ & CP Group & $(\mathrm{n}=478)$ & PValue \\
\hline Sex ratio, $\mathrm{M} / \mathrm{F}$ & 1.59 & 1.70 & & 0.64 \\
Age (years) & $58.79 \pm 13.28$ & $60.24 \pm 10.77$ & 0.35 \\
\hline
\end{tabular}

CP indicates clinical pathway. Values are mean \pm SD.

\section{LOS and Costs}

The average length of hospital stay was decreased from 16.70 days to 15.30 days after the implementation of clinical pathways with a statistical difference $(p<0.05$, Table 2$)$. The hospital costs were decreased from $\$ 2326.21$ to $\$ 2282.90$, but there was no significant difference between them $(p=$ 0.15 , Table 2). Specifically, the cost for drugs were reduced from $\$ 1144.64$ to $\$ 1045.54$ but the difference was not significant ( $p=0.19$, Table 2$)$; the inspection costs were significantly reduced from $\$ 422.80$ to 
$\$ 404.97(p<0.05$, Table 2); the nursing costs were significantly increased from $\$ 53.66$ to $\$ 56.76(p<$ 0.05 , Table 2).

\section{Discussion}

Prior to the development of clinical pathways supported by clinical data, our hospital attempted to alleviate patient costs and shorten hospital days in full compliance with the fixed clinical pathway. Unfortunately, this attempt didn't make a satisfactory achievement. The utilization rate of clinical pathway is low, and the mutation rate is high. This might be caused by the fact that our previous fixed clinical pathway templates limit our physicians' role in clinical decision-making. Therefore, based on evidence-based medicine, we used historical data to help clinicians build clinical pathway templates adapted to the actual situation of local hospitals, and developed a clinical pathway management system[8].

The LOS is an indicator of efficiency in the treatment of ischemic brain infarctions[10]. The clinical pathways application significantly shortened the LOS by 1.4 days (8.4\%) per cerebral infarction patient compared with control group. The main factors contributing to the amelioration of LOS varied and the biggest proportion of variance was explained by socio-demographic and clinical factors measured on patient level. Hospital equipment contributed somewhat additionally to the explanation of variance for hospitals specializing in internal and geriatric medicine. The clinical pathway standardizes the behavior of diagnosis and treatment, reduces unnecessary examination and inspection items and improves the efficiency of treatment, thus shortening the patient's time in hospital. The inspection costs reduction observed in the study might also benefit from the application of clinical pathway.

A previous study demonstrated the LOS, rescue, payment methods, gender, treatment outcomes and admissions were the main factors affecting hospital costs[11]. In this study, the average hospital costs decreased $\$ 43.91$ compared to without a clinical pathway, but there was no statistically significant $(\mathrm{P}=$ 0.15 , Table 2). Although, it was found that hospital stay was a major factor of hospital cost, and comprehensive measures should be taken to shorten the hospital costs[12]. Our findings do not yet well explain this view. This also illustrates the complexity of clinical processes and the difficulty of making clinical pathways.

Nowadays, there is no consistent and standardized nursing process to measure deviations in process or cost before we implement the clinical pathway for cerebral infarction treatment. Our new clinical pathways facilitate multidisciplinary communication, data collection, data analysis and feedback to suppliers. Clinical pathways are difficult to be designed and implemented because it is a highly interdisciplinary expertise involving knowledge and staff from various departments, thus resulting ini poor coordination and inefficient treatments.[13]. Therefore, scientific data support and optimized treatment strategies are critical for measuring and reengineering clinical pathways.

Discharge against medical advice from the hospital is an unneglectable issue from point of view of treatment management, health costs as well as the side effects of treatment [14]. As our results indicated, 
the rate of discharge against medical advice in our study was decreased dramatically from $7.9 \%$ to $4.4 \%$. Unfortunately, we were unable to get data on the patient or family satisfaction survey. However, the decline in the rate for discharge against medical advice might indirectly indicate that the patient's satisfaction has improved.

Although clinical pathways made a progressive achievement in our hospital, there are still some physicians who question the effects of clinical pathways and even worry about the excessive emphasis on variability will threaten their autonomy and limit their ability to cope with specific patients. However, after the implementation of our new clinical pathways, physicians are increasingly hugging clinical pathways because our new clinical pathways provide the best treatment options and optimize the process of diagnosis and treatment. Besides, physicians' decisions were incorporated into our new clinical pathways. Under some unexpected circumstances, physicians even have the discretion to go beyond the pathways and behave as they did without pathways.

Together, our clinical pathways might benefit more physicians and patients if there are more hospitals can employ this system. Considering the fact that medical resources vary significantly from metropolitan cities to rural counties in China, the more advanced clinical pathways should be established according to local medical resource status.

\section{Abbreviations}

clinical pathways (CP), the length of in-hospital stay (LOS)

\section{Declarations}

- Ethics approval and consent to participate

The experimental protocol was established, according to the ethical guidelines of the Helsinki Declaration and was approved by the Human Ethics Committee of Ethics Committee of Institute of Basic Research in Clinical Evaluation, China Academy of Chinese Medical Sciences.

- Consent to publish

Not applicable.

- Availability of data and materials

All data generated or analysed during this study are included in this published article.

- Competing interests

The authors declare that they have no competing interests.

- Funding 
National Key R\&D Program (2017YFC1703505);

- Authors' Contributions

All authors read and approved the final manuscript.

- Acknowledgements

Not applicable.

\section{References}

1. Every N R, Hochman J, Becker R, et al. Critical Pathways: A Review[J]. Circulation, 2003, 101(4):461465.

2. Rotter T, Kinsman L, James E L, et al. Clinical pathways: effects on professional practice, patient outcomes, length of stay and hospital costs[J]. International Journal of Evidence-Based Healthcare, 2011, 9(2):191-192.

3. He J A, Yang W. Clinical pathways in China-an evaluation[J]. International Journal of Health Care Quality Assurance, 2015, 28(4):394-411.

4. Norrving, B. Global stroke initiative[J]. Journal of the Neurological Sciences, 2017, 381:25.

5. Organization W H. World health report 2010[J]. Bmj, 2004, 328(7430):: 6.

6. Liu L, Wang D, Wong K S L, et al. Stroke and Stroke Care in China: Huge Burden, Significant Workload, and a National Priority[J]. Stroke, 2011, 42(12):3651-3654.

7. Katzan I L, Cebul R D, Husak S H, et al. The effect of pneumonia on mortality among patients hospitalized for acute stroke[J]. Neurology, 2003, 60(4):620.

8. Narayanan J, Dobrin S, Choi J, et al. Structured clinical documentation in the electronic medical record to improve quality and to support practice-based research in epilepsy[J]. Epilepsia, 2016.

9. Zhong W, Liu Y, Wu Z, et al. A Study on the Construction of Semi-structured Electronic Medical Records Based on Clinical Pathways[J]. Chinese Medical Record English Edition, 2013, 1(8):338342.

10. Tan G, Liu M, Lei C, et al. Influencing factors analysis of hemorrhagic transformation in nonthrombolysis patients after acute cerebral infarction[J]. Chinese Journal of Cerebrovascular Diseases, 2015, 12(8):409-414.

11. Wang X, Wang G, Wu J, et al. The Analysis on the Influencing Factors of Hospital Costs for Cerebral Infarction Patients[M]// Green Communications and Networks. 2012.

12. Unrath $\mathrm{M}$, Kalic M, Berger K. [Length of hospital stay of patients with ischemic brain infarction:, trends over 10 years and analysis of influencing factors][J]. Deutsche Medizinische Wochenschrift, 2012, 137(34-35):1683. 
13. Killander A J, Killander A J. Why design methodologies are difficult to implement[J]. International Journal of Technology Management, 2001, 21(3/4):271-276.

14. Pour Karimi S A, Saravi B M, Farahabbadi E B, et al. Studying the Rate and Causes of Discharge Against Medical Advice in Hospitals Affiliated to Mazandaran University of Medical Sciences[J]. Materia Socio Medica, 2014, 26(3):203-207.

\section{Tables}

Table 2. LOS and costs of the patients with cerebral infarction, 2014-2016

\begin{tabular}{llll}
\hline & Control Group (n=444) & CP Group & PValue \\
& & $(\mathrm{n}=478)$ & \\
\hline LOS (days) & $16.70 \pm 9.80$ & $15.30 \pm 8.07$ & 0.04 \\
Average Hospital Costs, \$ & $2326.21 \pm 1802.49$ & $2282.90 \pm 1642.37$ & 0.15 \\
Drug Costs, \$ & $1144.64 \pm 883.40$ & $1045.54 \pm 696.48$ & 0.19 \\
Inspection costs, \$ & $422.80 \pm 176.99$ & $404.97 \pm 205.75$ & 0.04 \\
Nursing costs, \$ & $53.66 \pm 90.50$ & $56.76 \pm 44.08$ & $<0.001$ \\
Discharge without doctor's advice & $35(7.9 \%)$ & $21(4.4 \%)$ & $<0.001$ \\
\hline
\end{tabular}

$\mathrm{CP}$ indicates clinical pathway. Values are mean \pm SD.

\section{Figures}


Between June 1, 2014 and May 31, 2016, 1533 patients with cerebra infarction were recognized and treated in the hospital. ( $n=1533$ )

Control group, June 1,2014 - May,31,2015, (n=733)

CP group, June 1, 2015 - May,31,2016, $(n=800)$
Excluded aged more than 80 yearsin $=273$ )

- Control group $(n=136)$

-CP group $(n=137)$

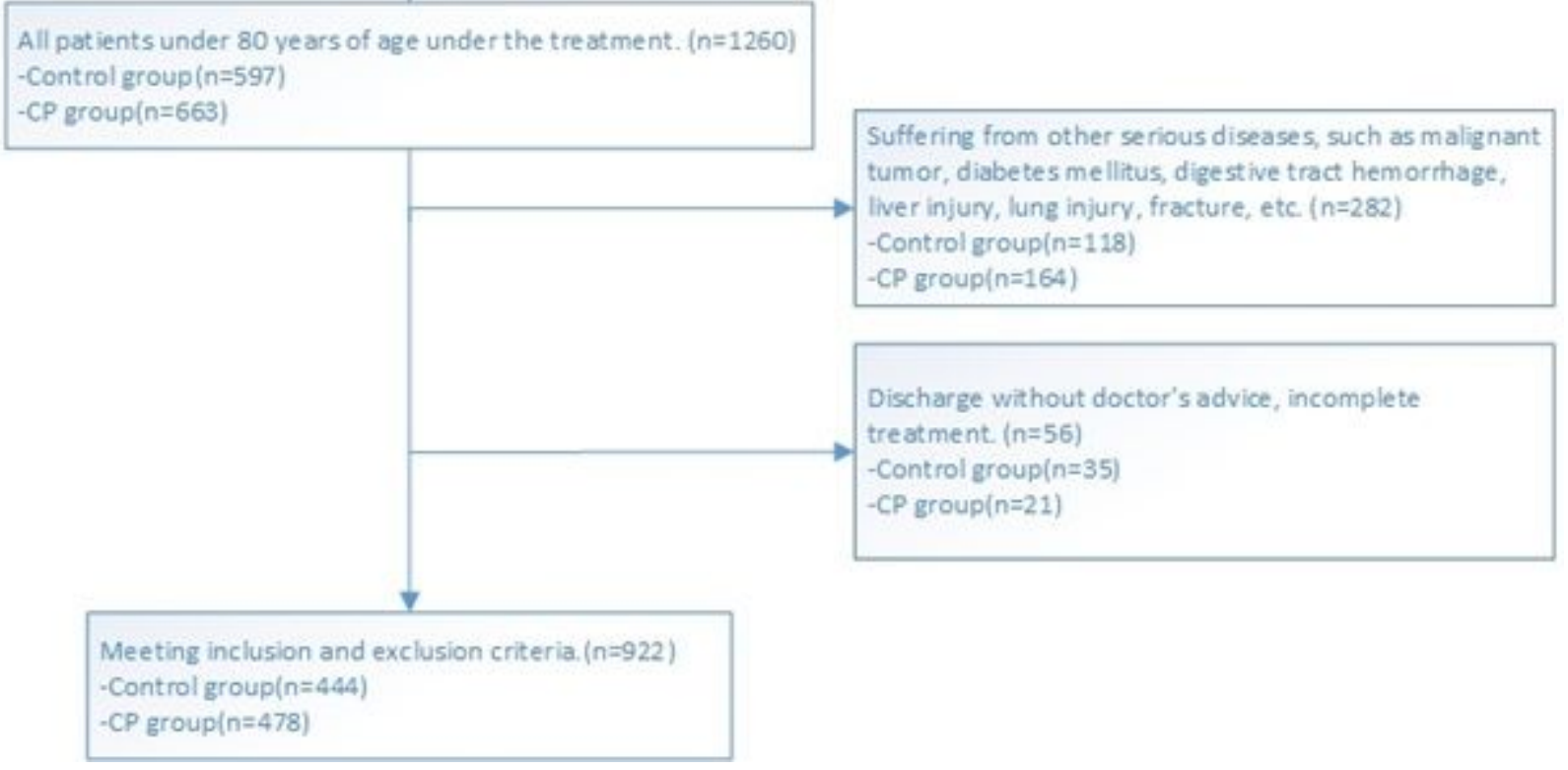

\section{Figure 1}

Flow chart of patients and hospital stays included in this study between June 1, 2014 and May 31, 2016 